\title{
Simultaneous Rupture of the Anterior Cruciate Ligament, Medial Collateral Ligament and Patellar Tendon: A Case Series, Review of the Literature, and Proposed Treatment Algorithm
}

\author{
Matthew Quinn, $B S^{1^{*}}$, Nicholas Lemme, $M D^{1}$, Anna King, $D O^{2}$ and Ramin Tabaddor, $M D^{1}$ \\ ${ }^{1}$ Warren Alpert Medical School, Brown University, USA \\ ${ }^{2} \mathrm{NYU}$ College of Medicine, USA
}

*Corresponding author: Matthew Quinn, MS-4, Warren Alpert Medical School, Brown University, 222 Richmond Street, Providence RI - 02903, USA, Tel: 401-369-2223

\begin{abstract}
Patellar Tendon (PT) rupture in combination with an anterior cruciate ligament injury $(A C L)+/-$ a Medial Collateral Ligament (MCL) injury is a rarely reported injury pattern. We report the cases of three athletes who sustained simul-taneous rupture of their PT, ACL and MCL while competing. They were treated in a staged manner with acute pri-mary repair of the patellar tendon and MCL followed by a rehabilitation period. Reconstruction of the ACL was performed once range of motion and strength of the extensor mechanism was sufficient. At one year follow up each patient had an IKDC score of $>80$ and a Lysholm score $>89$. There are now 29 reported cases of simultaneous rupture of the patellar tendon and ipsilateral ACL and 21 reported cases of simultaneous rupture of the patellar tendon, ipsilateral ACL, and MCL. There has been no consensus on management of these complex knee injuries. We review the literature and propose a treatment algorithm for the management of these multi-ligamentous knee injuries.
\end{abstract}

\section{Keywords}

Medial collateral ligament, Patellar tendon, Knee, Treatment, Rupture

\section{Introduction}

Rupture of the $A C L$ is very common with an incidence estimated at 100,000-200,000 ruptures per year in the United States [1]. Patellar tendon (PT) ruptures, however, are very rare. According to a study by Clayton and Court-Brown [2], of 2794 soft tissue injuries only $0.6 \%$ involved the PT. Even more rare is a simultaneous rupture of the anterior cruciate ligament $(\mathrm{ACL})$ and the ipsilateral PT. Literature regarding this is limited with only 26 cases of combined ACL and PT ruptures reported [3]. The Medial Collateral Ligament (MCL) is the most frequently injured ligament of the knee with associated injuries reported to be as high as $78 \%$ in grade III sprains $[4,5]$. There are 19 reported cases of a simultaneous rupture of the $\mathrm{ACL}$, medial collateral ligament and the PT in the literature [3]. We report the cases of a collegiate athlete and two high school athletes who suffered simultaneous ipsilateral rupture of the ACL, PT and $\mathrm{MCL}$ and review the literature. To our knowledge, there is no established treatment protocol for this type of combined injury. We offer a treatment algorithm to help address this complex issue.

\section{Case Report 1}

A 20-year-old Division III collegiate football player presented to us after sustaining an injury to his right leg while playing in a football game. During a play, his right leg was planted in a slightly flexed position and he was struck forcefully from the left side. He was initially evaluated at a nearby emergency department, given a referral for an MRI, and was instructed to follow up with an orthopedic surgeon. We evaluated him 5 days post

Citation: Quinn M, Lemme N, Anna King DO, Tabaddor RR (2019) Simultaneous Rupture of the Anterior Cruciate Ligament, Medial Collateral Ligament and Patellar Tendon: A Case Series, Review of the Literature, and Proposed Treatment Algorithm. Int J Sports Exerc Med 5:144. doi.org/10.23937/24695718/1510144

Accepted: September 23, 2019; Published: September 25, 2019

Copyright: (c) 2019 Quinn M, et al. This is an open-access article distributed under the terms of the Creative Commons Attribution License, which permits unrestricted use, distribution, and reproduction in any medium, provided the original author and source are credited. 
injury with an MRI performed 4 days post injury. Physical examination revealed a $3+$ effusion with a palpable defect of the patella tendon and associated patella alta. He was unable to perform a straight leg raise. Lachman and Pivot Shift tests were limited secondary to pain and swelling. Grade $3 \mathrm{MCL}$ insufficiency was present. He was stable to varus stress. There was significant ecchymosis along the medial aspect of the knee. Magnetic Resonance Imaging (MRI) revealed a full thickness tear of the proximal $A C L$, a full thickness tear of the distal tibial insertion of the superficial MCL with retraction and a full thickness rupture of the patella attachment off the PT with $2 \mathrm{~cm}$ of retraction.

The patient was taken to the operating room two days after initial evaluation for a staged repair. An arthroscopy of the knee was first performed. The $A C L$ was confirmed to be ruptured off the femoral insertion. The remaining $A C L$ stump was debrided. The medial and lateral menisci were found to be intact. The open portion was then performed. The PT was found to be completely disrupted off the inferior pole of the patella and retracted with disruption of the medial and lateral retinaculi. The PT was primarily repaired using \#5 fiberwire secured into the patella tendon in a Krakow fashion and the sutures passed through 3 drill holes in the patella from inferior to superior pole where they were tied and secured. The medial and lateral retinaculi were then repaired with \#1 vicryls. The range of motion was assessed and found to range from 0-100 degrees without tension. The MCL was assessed and demonstrated complete disruption off of the tibial insertion. A two-anchor repair was performed.Postoperatively, the patient was placed in a knee immobilizer locked in extension and was allowed to weight bearing as tolerated. He was instructed to perform straight leg raises, calf pumps, and quadriceps sets daily. At 2 weeks post-operative, formal physical therapy was initiated and his flexion was progressed 15 degrees per week to 90 degrees for a period of six weeks. At 2 months postoperative his range of motion was 0-125 degrees with 4/5 quadriceps function, normal patellar tracking, and without evidence hyperextension or instability to valgus stress.

At 3 months postoperative, the patient returned to the operating room for $A C L$ reconstruction with a bonePT-bone allograft and assessment of the MCL. Although autograft was presented to patient as optimal graft type, the patient preferred to use allograft in order to avoid any comorbidities associated with autograft harvest on the contrala-teral side. Under anesthesia, the $\mathrm{ACL}$ graft was placed and the MCL was found to have excellent integrity. The medial and lateral menisci were again found to be normal to inspection. He was placed on a standard postoperative ACL protocol that focused on early ROM exercises with, establishing a normal gait pattern, gradually building strength, and eventually progressing towards sports specific activities in a step-wise fashion. At 1 year follow up, physical exam demonstrat- ed negative Lachman and ante-rior drawer tests with full strength and range of motion when compared to the contralateral side with a negative Lachman and anterior drawer test. He had an IKDC score of 80.5 and a Lysholm score of 90.

\section{Case Report 2}

A 15-year-old male football player presented with a left knee injury that occurred while being tackled in practice. With his $L$ foot planted, he was hit head on and from his right side simultaneously. He was initially managed by a primary care team and received and MRI 8 days after his initial injury. Imaging revealed PT rupture, distal MCL rupture, ACL rupture, and tears of both the medial and lateral menisci. He presented to clinic 12 days after the initial injury with continued pain and swelling. His knee demonstrated a 2+ effusion with soft tissue swelling and ecchymosis along the medial aspect of the knee. There was a positive Lachman, significant tenderness along the medial collateral ligament, and 3+ gapping with valgus stress. Additionally, the patient had a palpable PT defect with loss of extensor mechanism function.

The patient was taken to the operating room 3 days after presentation to clinic for staged repair. Using an arthroscopic approach, the ACL stump was located and debrided. The lateral meniscus was explored showed a stable radial tear in the posterior horn. The medial meniscus showed a vertical tear in the red-red zone extending from the anterior to mid body and was repaired. The posterior horn and body were intact. A linear incision was then made from the inferior pole of the patella to the distal portion of the tibial tubercle. The $\mathrm{MCL}$ was then identified and showed complete distal rupture. The PT was visualized and two \#2 fiber wires were whipstitched through the distal and proximal PT stumps in a Krakow fashion. The repair was then over sutured with another \#2 Fiberwire. The knee was taken through range of motion and the PT was found to be stable to approximately 100 degrees of flexion. The $\mathrm{MCL}$ was then repaired using four Suture Taks. The knee still demonstrated $1+$ gapping in 20 degrees of flexion. An internal brace augmentation repair was performed using Biocomposite Swive locks to create a double row repair of the $\mathrm{MCL}$. The knee was again taken through range of motion and was stable to valgus stress. Postoperatively, the patient was placed in a knee immobilizer locked in extension and was allowed to weight bearing as tolerated. He was instructed to perform straight leg raises, calf pumps, and quadriceps sets daily. He began formal physical therapy on postoperative day 10. At 2 weeks postoperative, formal physical therapy was initiated and his flexion was progressed 15 degrees per week to 90 degrees for a period of six weeks.

At 3 months post-operative, the patient had 0-120 degrees of motion at the knee with 4/5 strength when compared to the contralateral side. The knee was stable 
to varus stress, but demonstrated prominent gapping with valgus stress when compared to the contralateral side. Lachman's test remained positive. At this time the patient returned to the operating room for $A C L$ reconstruction using a Quadriceps Tendon (QT) autograft. It was conveyed to the patient that semitendinosus (ST) autograft would be the optimal graft type, but he preferred to proceed with QT given the involvement of the ipsilateral ST in his initial injury and his desire to avoid bilateral knee pain postoperatively. Under anesthesia, the QT was exposed and marked. The QT graft was then harvested and placed on tension. The medial and lateral meniscus demonstrated grade $0-1$ changes. ACL graft was then placed and was without evidence of impingement in extension or flexion. Lachman was performed and found to be negative. Postoperatively, he was placed on standard ACL rehabilitation protocol. At one year follow up, there was full and symmetric strength and ROM about the knees bilaterally. Lachman and anterior drawer tests were negative. The knee was stable to valgus and varus stress testing in extension and 20 degrees of flexion. IKDC score was 82.8 and Lysholm score was 90.

\section{Case Report 3}

A 17-year-old male lacrosse player presented with left knee pain after sustaining an injury during a lacrosse game. He stated that he collided with another player, his knee "buckled", and the other player subsequently landed on his knee as they both fell to the ground. He received $x$-rays the day of the injury which revealed questionable patella alta and possible PT rupture from the tibial tubercle. He was evaluated in clinic two days later. At that time, physical exam was significant for diffuse soft swelling from the mid-thigh down to the mid leg with a $3+$ effusion. Tenderness over the medial and lateral joint lines was present. Lachman was grossly positive, there was $2+$ gapping to valgus stress, and he had tenderness along the distal portion of the MCL. He was unable to activate his quadriceps and extend his knee while simultaneously having a palpable defect of his PT. He was sent for STAT MRI at this time which revealed a complex tear of the lateral meniscus, partial avulsion of the $\mathrm{MCL}$, rupture of the $\mathrm{ACL}$, and rupture of the PT.

The patient was taken to the operating room four days later for repair using a staged approach. Using an arthroscopic approach the ruptured $\mathrm{ACL}$ was identified and debrided. The medial meniscus was intact but there was significant damage to the medial capsule and retinaculum. The lateral meniscus demonstrated a complex tear of the posterior body and underwent meniscectomy. The remainder of the arthroscopic exploration did not require additional intervention. A longitudinal incision was then made, and the PT was identified and carefully dissected. The PT was repaired and secured into the tibia using \#2 Fiberwires in a Krakow fashion and a Bio Composite Swive Lock. The medial retinaculum was repaired using \#1 Vicryl. The MCL was then debrided to clean edges and a $2.4 \mathrm{~mm}$ BioComposite SutureTak was placed at the distal edge and the MCL was secured to its tibial insertion. The knee was taken through range of motion and demonstrated excellent stability to valgus stress. He was instructed to perform straight leg raises, calf pumps, and quadriceps sets daily. At 2 weeks post-

Table 1: Documented cases of PT + ACL +/- MCL Injury.

\begin{tabular}{|c|c|c|c|c|c|}
\hline Study & Patients & Injury Type & Delay of Diagnosis & MRI & Repair \\
\hline Baker, et al. [6] & Patient 1 & ACL/PT/MCL & PT (found at surgery) & $\mathrm{N}$ & $\begin{array}{l}\text { Primary PT repair with no MCL repair/ ACL } \\
\text { reconstruction }\end{array}$ \\
\hline Rae, et al. [7] & Patient 1 & ACL/PT/MCL & PT & $\mathrm{N}$ & $\begin{array}{l}\text { Primary repair PT/MCL with no } A C L \\
\text { reconstruction }\end{array}$ \\
\hline \multirow[t]{6}{*}{ Levakos, et al. [9] } & Patient 1 & ACL/PT/MCL & PT & $\mathrm{N}$ & $\begin{array}{l}\text { Single stage repair (primary PT/MCL repair, } \\
A C L \text { with allograft) }\end{array}$ \\
\hline & Patient 2 & ACL/PT & ACL (found postop) & $\mathrm{N}$ & $\begin{array}{l}\text { Single stage repair (primary PT repair the } \\
\text { ACL with ITB autograft } 3 \text { years later) }\end{array}$ \\
\hline & Patient 3 & ACL/PT & PT (found at surgery) & $\mathrm{N}$ & $\begin{array}{l}\text { Single stage repair (primary PT repair, ACL } \\
\text { with STG autograft) }\end{array}$ \\
\hline & Patient 4 & ACL/PT/MCL & None & $\mathrm{Y}$ & $\begin{array}{l}\text { Single stage } \mathrm{PT} / \mathrm{MCL} \text { repair with no } \mathrm{ACL} \\
\text { reconstruction }\end{array}$ \\
\hline & Patient 5 & ACL/PT/MCL & $\begin{array}{l}\text { ACL/MCL found } 1 \\
\text { year postop }\end{array}$ & $\mathrm{N}$ & $\begin{array}{l}\text { Single stage repair (primary } \mathrm{PT} \text { repair with } \\
\text { no } \mathrm{MCL} \text { repair/ACL reconstruction) }\end{array}$ \\
\hline & Patient 6 & ACL/PT/MCL & None & $Y$ & $\begin{array}{l}\text { Staged repair (primary PT/MCL repair then } \\
\text { ACL with allograft } 3 \text { months later) }\end{array}$ \\
\hline Chiang, et al. [22] & Patient 1 & ACL/PT & None & $\mathrm{Y}$ & $\begin{array}{l}\text { Single stage repair (primary PT repair, ACL } \\
\text { with STG autograft) }\end{array}$ \\
\hline \multirow[t]{2}{*}{$\begin{array}{l}\text { Costa-Paz, et al. } \\
{[10]}\end{array}$} & Patient 1 & ACL/PT/MCL & None & $Y$ & $\begin{array}{l}\text { Staged repair (primary PT/MCL repair, then } \\
\text { ACL with contralateral BTB autograft) }\end{array}$ \\
\hline & Patient 2 & ACL/PT/MCL & PT & $Y^{*}$ & $\begin{array}{l}\text { Single Stage repair (primary PT/MCL repair, } \\
\text { ACL with QT autograft) }\end{array}$ \\
\hline
\end{tabular}




\begin{tabular}{|c|c|c|c|c|c|}
\hline & Patient 3 & ACL/PT & None & $\mathrm{Y}$ & $\begin{array}{l}\text { Single stage repair (primary PT repair, ACL } \\
\text { with STG autograft) }\end{array}$ \\
\hline Chow, et al. & Patient 1 & ACL/PT & None & $\mathrm{N}$ & Primary PT repair with no ACL reconstruction \\
\hline Futch, et al. [25] & Patient 1 & ACL/PT & None & $\mathrm{Y}$ & $\begin{array}{l}\text { Single stage repair (primary } \mathrm{PT} \text { repair, } \mathrm{ACL} \\
\text { with BTB allograft) }\end{array}$ \\
\hline $\begin{array}{l}\text { Koukoulias, et al. } \\
\text { [8] }\end{array}$ & Patient 1 & ACL/PT/MCL & $A C L$ & $\mathrm{~N}$ & $\begin{array}{l}\text { Two stage repair, PT then STG autograft } \\
\text { ACL } 6 \text { months post op. }\end{array}$ \\
\hline Tsarouhas & Patient 1 & ACL/PT/MCL & $\begin{array}{l}\mathrm{ACL} \text { and } \mathrm{MCL} \text { found } \\
\text { at surgery }\end{array}$ & $\mathrm{N}$ & $\begin{array}{l}\text { Two stage repair, PT then STG autograft } \\
\text { ACL } 3 \text { months post op. }\end{array}$ \\
\hline Wissman & Patient 1 & $\begin{array}{l}\text { ACL/PT (high } \\
\text { grade partial) }\end{array}$ & None & Y & STG autograft ACL repair only \\
\hline \multirow[t]{3}{*}{ Mariani } & Patient 1 & ACL/PT/MCL & None & $\mathrm{Y}$ & $\begin{array}{l}\text { Two stage repair, PT then STG autograft } \\
\text { ACL once full ROM had been established. }\end{array}$ \\
\hline & Patient 2 & ACL/PT/MCL & None & $\mathrm{Y}$ & $\begin{array}{l}\text { Two stage repair, PT then STG autograft } \\
\text { ACL once full ROM had been established. }\end{array}$ \\
\hline & Patient 3 & ACL/PT/MCL & None & $\mathrm{Y}$ & $\begin{array}{l}\text { Two stage repair, PT then STG autograft } \\
\text { ACL once full ROM had been established. }\end{array}$ \\
\hline Gulabi & Patient 1 & ACL/PT/MCL & None & $\mathrm{Y}$ & $\begin{array}{l}\text { Single stage repair (primary PT repair, ACL } \\
\text { with STG autograft) }\end{array}$ \\
\hline Kim & Patient 1 & ACL/PT/MCL & None & $\mathrm{Y}$ & Single stage, allograft (achilles tendon) \\
\hline \multirow[t]{2}{*}{ Brunkhorst } & Patient 1 & ACL/PT/MCL & None & $\mathrm{Y}$ & $\begin{array}{l}\text { Two stage, } \mathrm{PT} \text { then hamstring autograft } \mathrm{ACL} \\
4 \text { months after index }\end{array}$ \\
\hline & Patient 2 & ACL/PT/MCL & None & $\mathrm{Y}$ & $\begin{array}{l}\text { Single stage, PT done with ACL to be done } \\
\text { on } f / u\end{array}$ \\
\hline \multirow[t]{2}{*}{ Cucchi, et al. [3] } & Patient 1 & ACL/PT/MCL & None & Y & $\begin{array}{l}\text { Single stage repair (primary PT repair, ACL } \\
\text { with STG autograft) }\end{array}$ \\
\hline & Patient 2 & ACL/PT/MCL & None & $\mathrm{Y}$ & $\begin{array}{l}\text { Single stage repair (primary PT repair, ACL } \\
\text { with STG autograft) }\end{array}$ \\
\hline \multirow[t]{3}{*}{ Current Author } & Patient 1 & ACL/PT/MCL & None & $\mathrm{Y}$ & Two stage repair, PT then BTB allograft ACL \\
\hline & Patient 2 & ACL/PT/MCL & None & $\mathrm{Y}$ & Two stage repair, PT then QT autograft ACL \\
\hline & Patient 3 & ACL/PT/MCL & None & $\mathrm{Y}$ & Two stage repair, PT then ST allograft ACL \\
\hline
\end{tabular}

ACL: Anterior Cruciate Ligament, BTB: Bone-Patellar Tendon-Bone, MCL: Medial Collateral Ligament, PT: Patellar Tendon, STG: Semitendonosis- Gracilis, QT: Quadriceps Tendon.

${ }^{*} \mathrm{MRI}$ not done immediately.

operative, formal physical therapy was initiated and his flexion was progressed 15 de-grees per week to 90 degrees for a period of six weeks. The patient continued with formal thera-py progressing towards restoration of full knee flexion and improvement of knee strength.

At 6 months post-operative the patient had achieved satisfactory strength and stability from physical therapy, and he returned to the operating room for $A C L$ repair with ST allograft. It was conveyed to the patient that semitendinosus (ST) autograft would be the optimal graft type, but he preferred to proceed with allograft given the involvement of the ipsilateral ST in his initial injury and his desire to avoid any potential comorbidities related to graft harvest on the contralateral side. The knee was arthroscopically explored and showed healed segments of the lateral meniscus and an intact medial meniscus. The graft was placed and secured using an Endobutton for the femoral component and a Biocomposite screw for the tibial component. The graft demonstrates good stability with probing, full range of motion, and stability with Lachman and anterior drawer testing. The patient was placed in a knee immobilizer and began physical therapy approximately 10 days later. At 15 month follow up the patient demonstrated full and symmetric strength and range of motion bi-laterally. Lachman and anterior drawer tests were negative and the knee was stability to valgus and varus stress. IKDC and Lysholm at this time were 83.9 and 89 , respectively.

\section{Discussion}

Overall, there are now 29 cases reported in the literature describing a PT rupture with an $A C L$ rupture +/- an MCL rupture (Table 1). Involvement of all three is highly uncommon with only 22 reported cases in the literature [3]. Baker [6] was the first to report this pattern of injury in 1980 on a 24-year-old basketball player who sustained a non-contact injury to the knee while coming down with a rebound. The PT rupture was identified at the time of surgery. The PT was primarily repaired but the $M C L$ and $A C L$ were left alone as they were felt to be irreparable. In 1991 Rae and Davies [7] reported on a 25-year woman who sustained a combined PT, ACL, and MCL injury while on a tram- 
poline. The diagnosis of PT rupture was initially missed and discovered at the time of surgery. The PT and $\mathrm{MCL}$ were repaired but the ACL was left unrepaired. The decision to repair the $\mathrm{MCL}$ acutely was based on greater than $10 \mathrm{~mm}$ medial joint line opening to valgus stress. They did not discuss the reason for not reconstructing the ACL. Additionally, Koukoulias, et al. [8] described a 47-year-old male who sustained a rupture of his $\mathrm{PT}, \mathrm{ACL}$, and $\mathrm{MCL}$. The diagnosis of $\mathrm{ACL}$ rupture was missed during initial evaluation and was discovered at the time of surgery. The PT and ACL were repaired in a staged approach. Tsarouhas, et al. treated a 38-yearold male that sustained a direct kick to the knee and presented with obvious PT deformity on examination. $M R I$ was not obtained and tears of the $A C L, M C L$, and lateral meniscus were found at the time of surgery. This case was also managed in a staged approach with acute PT repair followed by ACL repair [3]. Levakos, et al. [9] reported 6 cases of combined PT and ligamentous injuries. Four of those patients had simultaneous $\mathrm{PT}, \mathrm{ACL}$ and $\mathrm{MCL}$ injuries. The remaining 2 had PT and $A C L$ ruptures without involvement of the $M C L$, which were the first of its kind to be reported. Of the patients with combined PT, ACL and MCL injuries, the first was a 36-year-old who injured his knee while playing soccer. The PT rupture was initially missed and found at the time of surgery. The PT and the MCL were repaired and the $A C L$ was reconstructed during the same procedure. The second patient was a 20-year-old male who injured his knee while playing football. The PT and $\mathrm{MCL}$ were repaired. The $A C L$ was left alone and postoperatively wore a functional $A C L$ brace. The third patient was a 23-year-old male injured during a motorcycle accident. The PT was repaired acutely at an outside hospital without knowledge of the $A C L$ and $M C L$ injuries. One year later when referred to the author's facility for residual knee stiffness, an MRI was performed to assess the PT. An ACL and MCL tear were discovered. Both tears, given their chronic nature, were left alone and monitored. The last patient was a 23-year-old male who injured his knee during a football game. He was taken acutely to the operating room for a PT and $\mathrm{MCL}$ repair. $A C L$ was reconstructed 12 weeks later. Of these six patients, 2 received MRIs while the other 4 received no advanced imaging. Of the 4 that did not receive imaging, each of them had a missed diagnosis of either PT or ACL rupture. These cases represent instances in which significant injuries were missed due to the lack of advanced imaging in the setting of significant knee trauma.

Costa-Paz, et al. [10] reported 3 cases of combined PT and ligamentous injuries, 2 of which were simultaneous $\mathrm{PT}, \mathrm{ACL}$ and $\mathrm{MCL}$ injuries. The third case was a PT and ACL injury. The first case was a 31-year-old man injured during a motorcycle accident. The PT and $\mathrm{MCL}$ were repaired acutely. The patient later returned in a staged fashion 6 weeks later for ACL reconstruction. The second patient was a 31-year male who injured his knee while playing soccer. An ACL and $\mathrm{MCL}$ tear were clinically diagnosed at an outside hospital. The patient presented to the author's hospital 20 days later and was found to have no extensor mechanism. An MRI confirmed a PT rupture in addition to $M C L$ and $A C L$ tears. The PT and $M C L$ were repaired and the $A C L$ was reconstructed at the same surgery.

There are 7 reported cases of simultaneous PT and $A C L$ ruptures without MCL injury [3]. There are 2 cases, both reported by Levakos, et al. [9] in which the diagnosis of an injury was delayed. In one case, there was a delay of diagnosis of the PT which was discovered at the time of surgery. In the second case, the PT was repaired acutely in a 15-year-old female long jumper. After cast immobilization was removed 6 weeks later, the knee was found to be unstable and $A C L$ deficiency was diagnosed. The ACL was reconstructed 3 years later after the patient opted for non-operative treatment initially.

Of the 29 cases of simultaneous injuries to the PT and $A C L$ with and without $M C L$ injury, 9 cases involved delayed diagnosis of an injury (Table 1). Interestingly, only one of those patients had an MRI performed and that patient had an MRI 20 days after injury with an initial missed diagnosis of a PT rupture [10]. The 20 cases in which an MRI was obtained acutely had no missed injuries. This speaks to the diagnostic value of an MRI in an injury of this magnitude when clinical exams are limited from soft tissue swelling and patient discomfort. A missed diagnosis of a tendinous or ligamentous injury in the context of a multi-ligamentous injury or a combined ligament and tendon injury can alter treatment plan and adversely determine patient outcome. Given the severity of injury our patients, it was difficult to evaluate for concomitant injuries due to the degree of swelling and pain. This uncertainty and the ramifications of misdiagnosis lead us to pursued immediate MRI if it had not already been obtained. In the case of the third patient, this protocol likely prevented a delay in diagnosis of PT rupture. We would recommend immediate MRI evaluation for severe knee injuries in which a clinical exam is indeterminate, or the exam is suspicious for multiple injuries which is supported by the literature we have cited.

There is no consensus on the treatment of this rare simultaneous injury pattern whether it is a PT rupture with an $A C L$ and $M C L$ injury or with an $A C L$ injury alone. In the two cases of simultaneously injured PT, ACL and $M C L$ reported by Costa-Paz, et al. [10], one was a staged repair in which the PT and $\mathrm{MCL}$ were primarily repaired followed at a later time by an $\mathrm{ACL}$ reconstruction. The other case was treated with a single stage repair of all three injuries. Levakos, et al. [9] reported 4 cases of simultaneous injury to the PT, ACL and MCL. Each case was managed differently ranging from primary repair of the PT and $\mathrm{MCL}$ with no $\mathrm{ACL}$ reconstruction, primary re- 
pair of the PT with no MCL or ACL repair, single stage repair of all three injuries to a staged treatment of primary repair of the $\mathrm{PT}$ and $\mathrm{MCL}$ with $\mathrm{ACL}$ reconstruction three months later. Rae, et al. [7] managed their case with primary repair of the PT and MCL without ACL reconstruction. Lastly, Baker [6], who was the first to report a simultaneous injury of the PT, ACL and $\mathrm{MCL}$ treated his patient with PT repair only.

It is agreed that the PT should be repaired immediately to re-establish the extensor mechanism, re-establish leg control, and begin working towards restoring ROM [11-15]. Controversy, however, exists regarding the treatment of combined $\mathrm{ACL}$ and $\mathrm{MCL}$ injuries. There are authors who have recommended surgical treatment of both ligaments while others recommend operative management of the $\mathrm{ACL}$ and non-operative man- agement of the MCL [4,5,15-22]. However, one must consider that at least some of these protocols are established based on simultaneous $\mathrm{ACL}$ and $\mathrm{MCL}$ ruptures in the absence of a PT rupture. There is only one documented case of a simultaneous PT and $\mathrm{MCL}$ rupture which occurred in a recreationally active 45-year-old male. The injury was successfully managed with simultaneous repair of both PT and MCL with pain free resumption of normal activity levels at 18 months [23]. We propose that evaluation and surgical decision making for the MCL should be done at the time of the primary PT repair. If there is a complete avulsion or $>2+$ opening to valgus stress on exam, the MCL should be repaired acutely. We support this approach because of the improved likelihood of satisfactory function with surgical repair and the accessibility of the $M C L$ when using an open surgical technique. A 2017 review of current liter-

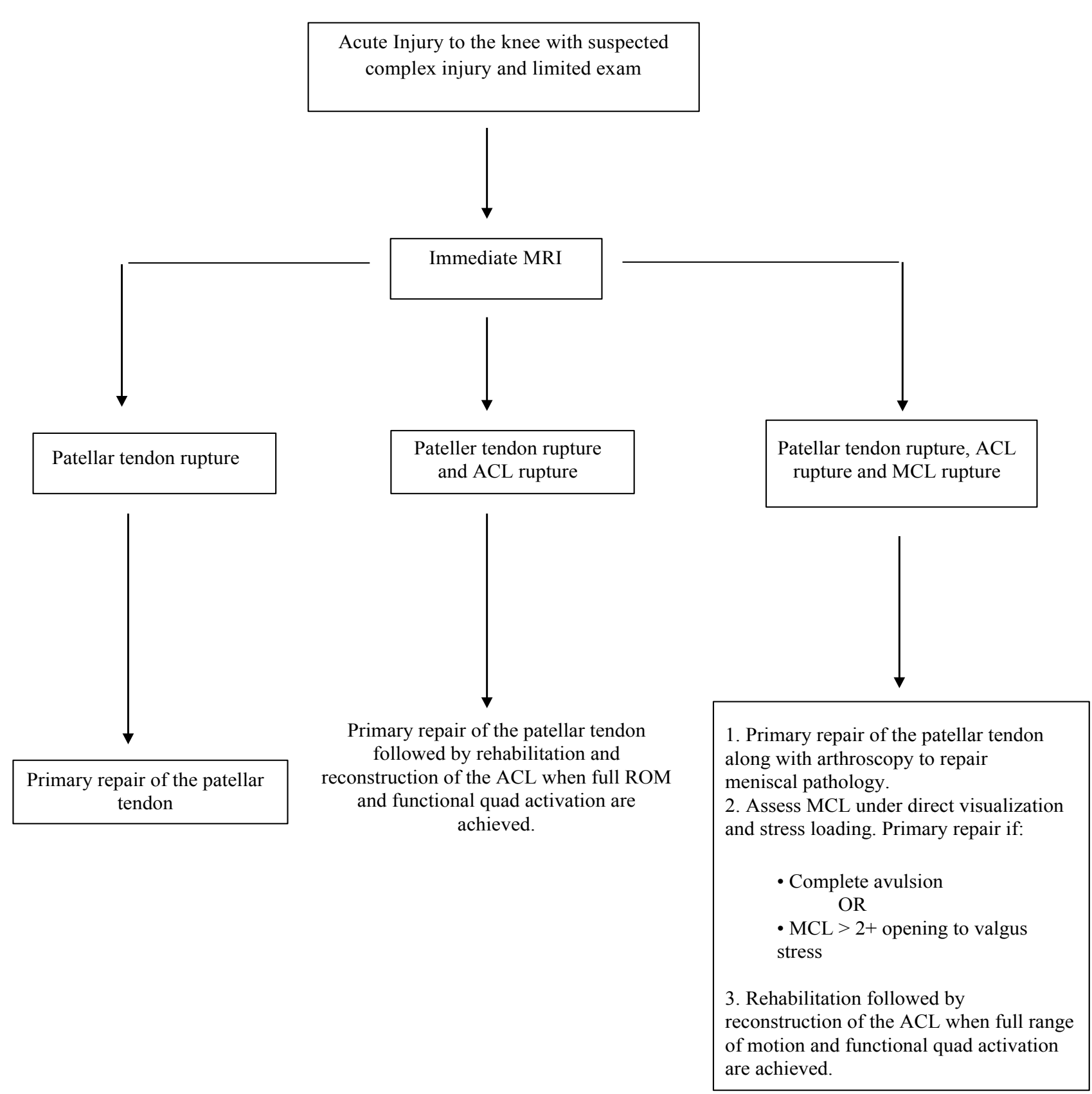

Figure 1: Proposed Treatment Algorithm 
ature by Moatshe, et al. [24] established the superiority of a surgical repair of torn ligaments in the setting of a multi-ligament knee injury. Their conclusion was based on data using patient reported outcomes, range of motion, Lysholm scores, contracture instability, and return to activity. Each of the outcomes were improved in surgical groups when compared to non-surgical groups. Additionally, by performing the MCL repair at the time of the PT repair the surgeon is able to assess the extent of injury to the $\mathrm{MCL}$ under direct visualization. The open approach also provides a convenient means of accessing the MCL for repair as opposed to using an arthroscopic approach during the $\mathrm{ACL}$ reconstruction.

It is important to note the benefits of a staged approach. Immediate repair of the PT diminishes the risk of quadriceps deactivation, persistent quadriceps atrophy, and most authors recommend a 6-week post-operative period where the knee is gradually re-introduced to flexion while still braced. Conversely, the standard ACL rehabilitation protocol emphasizes the gradual restoration of knee range of motion, closed chain exercises, and weight bearing as tolerated in the immediate post-operative phase of rehabilitation [25]. If the rehabilitation period for $A C L$ reconstruction is delayed, the risk of loss of function and development of arthrofibrosis increases $[8,25]$. By delaying reconstruction of the $A C L$, the patient is able to appropriately rehabilitate the PT repair, assure adequate range of motion, control swelling and reduce the overall risk for arthrofibrosis when compared to a single stage repair combined procedures may increase the risk of rehabilitation-related complications as arthrofibrosis, which may require repeated closed manipulation or arthroscopic lysis of adhesions [3]. Futch, et al. [25] performed a single stage procedure with primary repair of the PT and reconstruction of the $\mathrm{ACL}$. Their patient returned to the operating room at 4 months for lysis of adhesions and a manipulation under anesthesia. The patient returned 16 days later for another manipulation under anesthesia. Levakos, et al. [6] reports that one of their 3 patients who underwent a single stage repair of the PT and ACL had a $15^{\circ}$ loss of flexion at 18 months postoperative. They believed that arthrofibrosis contributed to this.

Regardless of one's preference in management, it is important to have a systematic and consistent approach to the evaluation of patients with a potentially complex injury pattern. Though this particular injury pattern is uncommon, it is possible that it is occurring with greater frequency than reported and is either missed or not reported. Our proposed treatment algorithm (Figure 1) starts with immediately obtaining an MRI within days after injury to determine the full extent of injury for aforementioned reasons. After clinical assessment and MRI review, the patient should be taken for immediate primary repair of the PT, repair or debridement of intrarticular pathology, and repair of the $\mathrm{MCL}$ if $>2+$ opening to valgus stress at $30^{\circ}$. The patient is allowed to rehabilitate per protocol for approximately 3 months to allow restoration of motion and diminishment of swelling. At this time, the patient should return for $A C L$ reconstruction using whichever graft type best suits their individual needs and preferences, most preferably a ST autograft. We would not recommend contralateral bone-PT-bone autograft as this may increase patient morbidity and could adversely affect rehabilitation with this particular injury pattern. This is followed by a standard ACL protocol. All three patients were found to have good results with this approach.

\section{Conclusion}

Simultaneous rupture of the $\mathrm{PT}, \mathrm{ACL}$, and $\mathrm{MCL}$ is a rare and easily missed pattern of injury. Prompt diagnosis is critical and early MRI can aide in detection of these injuries. There has been no consensus to treatment of this injury pattern. Our proposed treatment algorithm involves acquiring prompt MRI and employing a staged approach with immediate repair of the PT, repair of the $M C L$ based on severity of injury, and subsequent repair of the ACL once the PT has been appropriately rehabilitated.

\section{References}

1. Al-Khalifa FK, Alhamam NM, Uddin FZ, Aljawder AA, Abubaris RK, et al. (2014) Clinical outcomes following anterior cruciate ligament reconstruction utilizing hamstring tendon autografts. Saudi J Sports Med 14: 89-93.

2. Clayton R, Court-Brown C (2008) The epidemiology of musculoskeletal tendinous and ligamentous injuries. Injury 39 : 1338-1344.

3. Cucchi D, Aliprandi A, Nocerino E, Randelli P (2017) Early combined arthroscopic treatment for simultaneous ruptures of the patellar tendon and the anterior cruciate ligament leads to good radiological results and patient satisfaction. Knee Surg Sports Traumatol Arthrosc.

4. Fetto JF, Marshall JL (1978) Medial collateral ligament injuries of the knee. A rationale for treatment. Clin Orthop Relat Res 132: 206-218.

5. Miyamoto RG, Bosco JA, Sherman OH (2009) Treatment of medial collateral ligament injuries. J Am Acad Orthop Surg 17: 152-161.

6. Baker BE (1980) O'Donoghue's triad plus patellar tendon rupture. N Y State J Med 80: 1436-1437.

7. Rae PJ, Davies DR (1991) Simultaneous rupture of the ligamentum patellae, medial collateral, and anterior cruciate ligaments: A case report. Am J of Sports Med 19: 529-530.

8. Koukoulias N, Koumis $P$, Papadopoulos A, Kyparlis D, Papastergiou S (2011) Acute, simultaneous tear of patellar tendon and ACL: Possible mechanism of injury and rationality of the two-stage surgical treatment. BMJ Case Reports.

9. Levakos Y, Sherman M, Shelbourne D, Trakru S, Bonamo JR (1996) Simultaneous rupture of the anterior cruciate ligament and the patella tendon: six case reports. Am J Sports Med 24: 498-503.

10. Costa-Paz M, Muscolo DL, Mankino A, Ayerza MA (2005) Simultaneous acute rupture of the patellar tendon and the anterior cruciate ligament. Arthroscopy 21: 1143. 
11. Enad JG (1999) Patellar tendon ruptures. South Med J 92: 563-566.

12. Siwek CW, Rao JP (1981) Ruptures of the extensor mechanism of the knee joint. J Bone Joint Surg Am 63: 932-937.

13. Kasten P, Schewe B, Maurer F, Gösling T, Krettek C, et al. (2001) Rupture of the patellar tendon: a review of 68 cases and a retrospective study of 29 ruptures comparing two methods of augmentation. Arch Orthop Trauma Surg 121: $578-582$.

14. Capiola D, Re L (2007) Repair of patellar tendon rupture with suture anchors. Arthroscopy 23: 906.

15. Andersson C, Gillquist J (1992) Treatment of acute isolated and combined ruptures of the anterior cruciate ligament. A long-term follow-up study. Am J Sports Med 20: 7-12.

16. Hillard-Sembell D, Daniel DM, Stone ML, Dobson BE, Fithian DC (1996) Combined injuries of the anterior cruciate and medial collateral ligaments of the knee. Effect of treatment on stability and function of the joint. J Bone Joint Surg Am 78: 169-176.

17. Shelbourne KD, Porter DA (1992) Anterior cruciate ligamentmedial collateral ligament injury: nonoperatvie management of medial collateral ligament tears with anterior cruciate ligament reconstruction. A preliminary report. Am J Sports Med 20: 283-286.

18. Millett PJ, Pennock AT, Sterett WI, Steadman JR (2004) Early ACL reconstruction in combined ACL-MCL injuries. J Knee Surg 17: 94-98.
19. Noyes FR, Barber-Westin SD (1995) The treatment of acute combined ruptures of the anterior cruciate and medial collateral ligament of the knee. Am J Sports Med 23: 380389.

20. Schierl M, Petermann J, Trus P, Baumgartel F, Gotzen L (1994) Anterior cruciate and medial collateral ligament injury: ACL reconstruction and functional treatment of the MCL. Knee Surg Sports Traumatol Arthrosc 2: 203-206.

21. Indelicato PA, Linton R (2003) Medial ligament injuries in the adult, in Delee and Drez's Orthopaedics Sports Medicine: Principles and Practice. ( $2^{\text {nd }}$ edn), Philadelphia, PA: Saunders Publishing, 1937-1948.

22. Chiang AS, Shin SS, Jazrawi LM, Rose DJ (2005) Simultaneous ipsilateral ruptures of the anterior cruciate ligament and patellar tendon: a case report. Bull Hosp Jt Dis 62: 134-136.

23. De Baere T, De Muylder J, Deltour A (2014) An Unusual Knee Trauma: Combined Rupture of Medial Collateral Ligament and Patellar Tendon. Case Rep Orthop 2014: 657296

24. Moateshe G, Chahla J, Laprade R, Engebretson L (2017) Diagnosis and treatment of multiligament knee injury: State of the art. Journal of ISAKOS Joint Disorders and Orthopedic Sports Medicine 2.

25. Futch LA, Garth WP, Folsom GJ, Ogard WK (2007) Acute rupture of the anterior cruciate ligament and patellar tendon in a collegiate athlete. Arthroscopy 23: 112. 